\title{
Large Function Algebras with Certain Topological Properties
}

\author{
Artur Bartoszewicz and Szymon Głąb \\ Institute of Mathematics, Lodz University of Technology, Wólczańska 215, 93-005 Łódź, Poland \\ Correspondence should be addressed to Szymon Głąb; szymon.glab@p.lodz.pl
}

Received 30 October 2014; Accepted 14 January 2015

Academic Editor: Eva A. Gallardo Gutiérrez

Copyright (C) 2015 A. Bartoszewicz and S. Głąb. This is an open access article distributed under the Creative Commons Attribution License, which permits unrestricted use, distribution, and reproduction in any medium, provided the original work is properly cited.

Let $\mathscr{F}$ be a family of continuous functions defined on a compact interval. We give a sufficient condition so that $\mathscr{F} \cup\{0\}$ contains a dense $\mathfrak{c}$-generated free algebra; in other words, $\mathscr{F}$ is densely $\mathfrak{c}$-strongly algebrable. As an application we obtain dense $\mathfrak{c}$-strong algebrability of families of nowhere Hölder functions, Bruckner-Garg functions, functions with a dense set of local maxima and local minima, and nowhere monotonous functions differentiable at all but finitely many points. We also study the problem of the existence of large closed algebras within $\mathscr{F} \cup\{0\}$ where $\mathscr{F} \subset \mathbb{R}^{X}$ or $\mathscr{F} \subset \mathbb{C}^{X}$. We prove that the set of perfectly everywhere surjective functions together with the zero function contains a $2^{c}$-generated algebra closed in the topology of uniform convergence while it does not contain a nontrivial algebra closed in the pointwise convergence topology. We prove that an infinitely generated algebra which is closed in the pointwise convergence topology needs to contain two valued functions and infinitely valued functions. We give an example of such an algebra; namely, it was shown that there is a subalgebra of $\mathbb{R}^{\mathbb{R}}$ with $2^{c}$ generators which is closed in the pointwise topology and, for any function $f$ in this algebra, there is an open set $U$ such that $f^{-1}(U)$ is a Bernstein set.

\section{Introduction}

The algebraic properties of sets of functions have been considered in analysis for many years. One direction of such research is finding the so-called maximal (additive, multiplicative, and so on) classes for certain families of functions. For example, it was proved in [1] that the maximal additive class for Darboux real functions is the set of all constant functions. Recently, a new point of looking on the largeness of sets of functions has appeared. One can call a set $A$, contained in some algebraic structure of functions, a big one if $A$ (or $A \cup\{0\}$ ) contains a large, nice substructure inside. The first papers written in this direction were [2-4] and then [5-7]. In these papers, the notions contained in the following definition can be found.

Definition 1. Let $\kappa$ be a cardinal number.

(1) Let $\mathscr{L}$ be a vector space and let $A \subset \mathscr{L}$. We say that $A$ is $\kappa$-lineable if $A \cup\{0\}$ contains a $\kappa$-dimensional vector space.
(2) Let $\mathscr{L}$ be a Banach space and $A \subset \mathscr{L}$. We say that $A$ is spaceable if $A \cup\{0\}$ contains an infinite dimensional closed vector space.

(3) Let $\mathscr{L}$ be a commutative algebra and let $A \subset \mathscr{L}$. We say that $A$ is $\kappa$-algebrable if $A \cup\{0\}$ contains a $\kappa$ generated algebra $\mathscr{B}$ (i.e., the minimal cardinality of the set generating $\mathscr{B}$ equals $\kappa$ ).

Bartoszewicz and Głąb in [8] introduced the notion of strong algebrability.

Definition 2. Let $\kappa$ be a cardinal number, let $\mathscr{L}$ be a commutative algebra, and let $A \subset \mathscr{L}$. One says that $A$ is strongly $\kappa$-algebrable if $A \cup\{0\}$ contains a $\kappa$-generated free algebra.

Let us observe that the notion of spaceability is not a fully algebraic property but it has a topological ingredient (we ask about the existence of closed subspace of given Banach space). Ciesielski et al. in [9] asked about the existence of large linear subspaces, closed in the pointwise or uniform convergence 
topology in $\mathbb{R}^{\mathbb{R}}$ or $\mathbb{C}^{\mathbb{C}}$. So, following this way, one can define spaceability in linear topological spaces.

Some authors were interested in searching for a large substructure with some other topological property, namely, dense lineability (or algebrability) of some classes of functions. For example, Bayart and Quarta in [10] proved that the set $\mathcal{N} \mathscr{H}$ of all nowhere Hölder functions is densely $\omega$ algebrable in $C[0,1]$. In [11], Bastin et al. proved that the set of all nowhere Gevrey functions is densely c-algebrable in $C_{\infty}[0,1]$.

The aim of our paper is to formulate, prove, and apply some techniques of constructing dense $\mathfrak{c}$-generated free algebras in the space of continuous functions on a compact interval and to consider the possibility of the existence of closed algebras in some sets of real or complex functions.

\section{Dense Strong c-Algebrability in $C[0,1]$}

It is a simple observation that the set $\{x \mapsto \exp (r x): r \in \mathbb{R}\}$ is linearly independent in $\mathbb{R}^{\mathbb{R}}$. Moreover, if $X \subset \mathbb{R}$ is linearly independent over $\mathbb{Q}$, then $\{x \mapsto \exp (r x): r \in X\}$ is the set of free generators. In [12] the authors, using the composition of a function with some needed properties with such an exponential function, proved the $\mathfrak{c}$-algebrability of the set $C M m([0,1])$ of continuous functions with dense sets of local extrema. Recently, this idea has been further developed in $[13,14]$.

Let us call, after [13], a function $f: \mathbb{R} \rightarrow \mathbb{R}$ exponentiallike of rank $m$ whenever $f$ is given by the formula $f(x)=$ $\sum_{i=1}^{m} a_{i} \exp \left(\beta_{i} x\right)$ for some pairwise distinct nonzero numbers $\beta_{1}, \ldots, \beta_{m}$ and some nonzero numbers $a_{1}, \ldots, a_{m}$. We have the following.

Theorem 3 (see [13]). Let $\mathscr{F} \subseteq \mathbb{R}^{[0,1]}$ and assume that there exists a function $F \in \mathscr{F}$ such that $f \circ F \in \mathscr{F} \backslash\{0\}$ for every exponential-like function $f: \mathbb{R} \rightarrow \mathbb{R}$. Then $\mathscr{F}$ is strongly $\mathfrak{c}$-algebrable. More exactly, if $H \subseteq \mathbb{R}$ is a set of cardinality $\mathfrak{c}$ and linearly independent over the rationals $\mathbb{Q}$, then $\exp \circ(r F)$, $r \in H$, are free generators of an algebra contained in $\mathscr{F} \cup\{0\}$.

Using Stone-Weierstrass theorem, it is not difficult to observe that the algebra described in Theorem 3 is dense in $C[0,1]$ if and only if the function $F$ is continuous and strictly monotonic. This argument is described in the last section of [14]. To illustrate this, consider the following two examples. Let $\mathscr{F}$ stand for the set of all continuous functions which are differentiable $n-1$ times but not differentiable $n$ times at any point of their domains. Let $F$ be the $(n-1)$ th antiderivative of a strictly positive nowhere differentiable function. Then by [14, Theorem 4.5], the family $\mathscr{F}$ is densely $\mathfrak{c}$-strongly algebrable. In turn, using [14, Theorem 4.9] and a similar argument, one can prove that the set of all functions from $\mathscr{C}_{1}$, whose derivative is not $\alpha$-Hölder (for any $\alpha \in(0,1])$ at all but finitely many points, is densely $\mathfrak{c}$-strongly algebrable.

However, for many classes of functions, the monotonic representative does not exist. Here we propose some method of construction of a dense algebra even if $\mathscr{F}$ does not contain any monotonic function.
2.1. Nowhere Constant Continuous Functions. Let $F$ : $[a, b] \rightarrow \mathbb{R}$ be a continuous function. Then $F$ is called left nondecreasing at $x \in(a, b]$ if there is $\delta>0$ such that $F(y) \leq F(x)$ for any $y \in(x-\delta, x)$. Analogously we define a left nonincreasing function at $x \in(a, b]$ and right nondecreasing (nonincreasing) function at $x \in[a, b)$. We say that $x \in(a, b)$ is a point of local monotonicity, provided that $F$ is left nondecreasing or left nonincreasing and $F$ is right nondecreasing or right nonincreasing; see $[15,16]$. Note that if $x$ is a point of local minimum (local minimizer) or a point of local maximum (local maximizer) of $F$, then $x$ is a point of local monotonicity. We say that $F$ is nowhere constant, provided that its restriction to any open interval is not constant.

Fix a function $g \in C[\alpha, \beta]$ which is nowhere constant and such that $\alpha$ and $\beta$ are points of (one-sided) monotonicity of $g$. For $x \in[\alpha, \beta]$, denote by $H(x)$ the largest possible $y \in[x, \beta]$ such that $g(t)$ is between $g(x)$ and $g(y)$ for every $t \in[x, y]$ (here by $[x, x]$ we mean the singleton $\{x\}$ ). Such a number $H(x)$ always exists by the continuity of $g$. Let $x=H^{0}(x)$ and inductively $H^{n+1}(x)=H\left(H^{n}(x)\right)$ for $x \in[\alpha, \beta]$.

Lemma 4. Let $n \in \mathbb{N}$. If $H^{n+1}(\alpha)<\beta$, then

(i) $H^{n+1}(\alpha)>H^{n}(\alpha)$;

(ii) $H^{n+1}(\alpha)$ is a point of local extremum of $g$;

(iii) $H^{n+1}(\alpha)$ is a local minimizer of $g$ if and only if $H^{n}(\alpha)$ is a local maximizer of $g$.

Proof. Since $\alpha$ is a point of right local monotonicity of $g$, say $g$ is right nondecreasing at $\alpha$, then there is $\delta>0$ such that $g(\alpha) \leq g(y)$ for every $y \in(\alpha, \alpha+\delta)$. Let $t \in(\alpha, \alpha+\delta]$ such that $g(t)=\max \{g(y): y \in[\alpha, \alpha+\delta]\}$. Since $g$ is nowhere constant, then $g(t)>g(\alpha)$. Hence, $H(\alpha) \geq t>\alpha$.

Now, we will show that $H(\alpha)$ is a point of right local monotonicity of $g$. Suppose not, then by the definition of $H$, $g(y) \geq g(\alpha)$ for $y \in[\alpha, H(\alpha)]$. Moreover $g(H(\alpha))>g(\alpha)$. Let $\delta>0$ such that $|g(H(\alpha))-g(y)|<(g(H(\alpha))-g(\alpha)) / 2$ for $y \in(H(\alpha), H(\alpha)+\delta]$. Then $\left.g\right|_{[H(\alpha), H(\alpha)+\delta]}$ attains its maximum at some $w \in[H(\alpha), H(\alpha)+\delta]$. Since $g$ is not right nonincreasing at $H(\alpha)$, then $g(H(\alpha))<g(w)$. Moreover $g(\alpha) \leq g(y) \leq g(w)$ for any $y \in[\alpha, w]$. This contradicts the definition of $H$.

Proceeding inductively we obtain that $H^{n+1}(\alpha)>H^{n}(\alpha)$ or $H^{n+1}(\alpha)=\beta$. Note that $H^{1}(\alpha), H^{2}(\alpha), \ldots, H^{n}(\alpha)$ are local extrema of $g$. Moreover if $H^{i}(\alpha)$ is a local minimizer of $g$, then $H^{i+1}(\alpha)$ is a local maximizer of $g$ and vice versa.

Lemma 5. There is $n \in \mathbb{N}$ such that $H^{n}(\alpha)=\beta$.

Proof. Suppose that $H^{n}(\alpha)<\beta$ for every $n \in \mathbb{N}$. By Lemma 4 , the sequence $\left(H^{n}(\alpha)\right)_{n \in \mathbb{N}}$ is strictly increasing. Let $w=\lim _{n \rightarrow \infty} H^{n}(\alpha)$. If $g$ is left nondecreasing at $w$, then there is $\delta>0$ such that $g(y) \leq g(w)$ for every $y \in[w-\delta, w]$. Let $n$ be such that $H^{n}(\alpha)$ is a local minimizer of $g$ with $H^{n}(\alpha)>w-\delta$. Then for any $y \in\left(H^{n}(\alpha), w\right)$ we have $g\left(H^{n}(\alpha)\right) \leq g(y) \leq g(w)$ which contradicts the definition of $H^{n+1}(\alpha)$. In the same manner, we show that $g$ is not left 
nonincreasing. Therefore $w<\beta$, since $g$ is left monotonous at $\beta$.

Suppose now that $g$ is not right nondecreasing at $w$. Let $v \in[w, \beta]$ be a minimizer of $g$ on $[w, \beta]$. Then $v>w$ and $g(v)<g(w)$. Let $\delta>0$ be such that $|g(y)-g(w)|<$ $(g(w)-g(v)) / 2$ for every $y \in(w-\delta, w)$. Then fix $n \in \mathbb{N}$ such that $H^{n}(\alpha) \in(w-\delta, w)$ is a local maximizer of $g$ and $g\left(H^{k}(\alpha)\right) \leq g\left(H^{n}(\alpha)\right)$ for $k \geq n$. This is possible since $\lim _{k \rightarrow \infty} g\left(H^{k}(\alpha)\right)=g(w)<g\left(H^{n}(\alpha)\right)$ and $g$ is continuous. Therefore, $g\left(H^{n}(\alpha)\right) \geq g(y) \geq g(v)$ for $y \in\left(H^{n}(\alpha), v\right)$, which contradicts the definition of $H^{n+1}(\alpha)$. Similarly one can prove that the assumption that $g$ is not right nonincreasing at $w$ also leads to contradiction. Hence, $g$ is both right nondecreasing and right nonincreasing at $w$. This means that $g$ is constant on $[w, w+\delta]$ for some positive $\delta$, which contradicts the fact that $g$ is nowhere constant. This shows that $H^{n}(\alpha)=\beta$ for some $n \in \mathbb{N}$.

Lemma 6. Let $F \in C[a, b]$ be nowhere constant and $F(a) \leq$ $F(x) \leq F(b)$ for any $x \in[a, b]$. Let $\varepsilon>0$. Then there is $a$ partition $a=x_{0}<x_{1}<x_{2}<\cdots<x_{n}=b$ such that

(i) $F(x)$ is between $F\left(x_{k}\right)$ and $F\left(x_{k+1}\right)$ for $x_{k} \leq x \leq x_{k+1}$ and $k=0,1 \ldots, n-1$;

(ii) the mesh $\max \left\{x_{i+1}-x_{i}: i=0,1, \ldots, n-1\right\}$ of the partition is smaller than $\varepsilon$.

Proof. Let $a=t_{0}<t_{1}<t_{2}<\cdots<t_{m}=b$ be any partition of $[a, b]$ with the mesh smaller than $\varepsilon$. We will find a new partition $a=v_{0}<v_{1}<v_{2}<\cdots<v_{k}=b$ of $[a, b]$ such that each interval $\left[v_{i}, v_{i+1}\right)$ contains at most one $t_{j}$ and each $v_{i}$ is a point of local monotonicity of $F$. This new partition will also have a mesh smaller than $\varepsilon$. We construct it in the following way.

If $t_{i}$ is a point of local monotonicity of $F$, then $t_{i}$ remains in the new partition. Otherwise, by the fact that $F$ is nowhere constant the restriction $\left.F\right|_{\left[t_{i}-\varepsilon / 3, t_{i}\right]}$ attains its minimum at some $w_{L} \in\left[t_{i}-\varepsilon / 3, t_{i}\right]$ and maximum at some $w_{L}^{\prime} \in\left[t_{i}-\right.$ $\left.\varepsilon / 3, t_{i}\right]$. If one of the points $w_{L}, w_{L}^{\prime}$ is in $\left(t_{i}-\varepsilon / 3, t_{i}\right)$, then it is a point of local monotonicity and we put it to the new partition. However, it may happen that $\left\{w_{L}, w_{L}^{\prime}\right\}=\left\{t_{i}-\varepsilon / 3, t_{i}\right\}$; that is, $w_{L}$ and $w_{L}^{\prime}$ are the endpoints of the interval $\left[t_{i}-\varepsilon, t_{i}\right]$. We may assume that $w_{L}=t_{i}-\varepsilon / 3$ and $F\left(w_{L}\right)<F\left(t_{i}\right)$. Take any $t \in\left(t_{i}-\varepsilon / 3, t_{i}\right)$. If $t$ is a point of local monotonicity of $F$, then we are done. Assume now that $t$ is not a point of local monotonicity of $F$. This means that either $t$ is not a point of left monotonicity of $F$ or it is not a point of right monotonicity of $F$. We may assume that $t$ is not a point of left monotonicity of $F$. Then, $F$ attains its maximum on $\left[w_{L}, t\right]$ on some $w \in\left(w_{L}, t\right)$ and $w$ is a both-sided monotonicity point of $F$; $w$ is between $t_{i}-\varepsilon / 3$ and $t_{i}$, and we put it to the new partition. Similarly one can find an appropriate both-sided monotonicity point in $\left(t_{i}, t_{i}+\varepsilon / 3\right)$ which we put into the new partition.

In the next step we will find a refinement $a=x_{0}<x_{1}<$ $x_{2}<\cdots<x_{n}=b$ of $a=v_{0}<v_{1}<v_{2}<\cdots<v_{k}=b$ for which (i) holds true. To find such a refinement, for every $i<k$, we use Lemma 5 for the restriction $g=\left.F\right|_{\left[v_{i}, v_{i+1}\right]}, \alpha=v_{i}$, and $\beta=v_{i+1}$.

The assumption that $F$ is nowhere constant in Lemma 6 is essential. To see it, consider a function $F$ given by

$$
F(x)= \begin{cases}x, & x \in[1,2], \\ x \sin \left(\frac{\pi}{2 x}\right), & x \in(0,1), \\ 0, & x \in[-1,0], \\ 2 x+2, & x \in[-2,-1) .\end{cases}
$$

Note that $F(-2) \leq F(x) \leq F(2)$. For every partition $-2=x_{0}<$ $x_{1}<\cdots<x_{n}=2$ with the mesh smaller than 1 , there is the largest $k$ with $x_{k} \leq 0$. Then, $F\left(x_{k}\right)=0$ and we may assume that $F\left(x_{k+1}\right) \geq 0$. But there is $x \in\left(0, x_{k+1}\right)$ with $F(x)<0$, which means that the assertion of Lemma 6 does not hold for $F$. The problem is that $F$ is constant on $[-1,0]$.

Lemma 7. Let $E \subset \mathbb{R}$ be a finite set which is linearly independent over $\mathbb{Q}$. Let $F:[a, b] \rightarrow \mathbb{R}$ be a nowhere constant continuous function with $F(a) \leq F(x) \leq F(b)$ for any $x \in[a, b]$. Then, for any $\varepsilon>0$, there are $a=x_{0}<x_{1}<$ $x_{2}<\cdots<x_{n}=b$ and $F_{0} \in C[a, b]$ such that

(i) $\left\|F_{0}-i d\right\|_{\text {sup }}<\varepsilon$;

(ii) $F_{0}(x)=a_{k} F(x)+b_{k}$ for $x_{k} \leq x \leq x_{k+1}, k=0,1, \ldots, n-$ 1 ;

(iii) the set $\left\{a_{0}, a_{1}, \ldots, a_{n-1}\right\} \cup E$ is linearly independent over $\mathbb{Q}$.

Proof. By the previous lemma there are $a=x_{0}<x_{1}<x_{2}<$ $\cdots<x_{n}=b$ such that

(i) $F(x)$ is between $F\left(x_{k}\right)$ and $F\left(x_{k+1}\right)$ for $x_{k} \leq x \leq x_{k+1}$ and $k=0,1 \ldots, n-1$;

(ii) $\max \left\{x_{i+1}-x_{i}: i=0,1, \ldots, n-1\right\}<\varepsilon / 3$.

We can find real numbers $a_{0}, b_{0}$ such that the set $E \cup\left\{a_{0}\right\}$ is linearly independent over $\mathbb{Q}, a_{0} F\left(x_{0}\right)+b_{0}=x_{0}(=a)$, and $\left|a_{0} F\left(x_{1}\right)+b_{0}-x_{1}\right|<\varepsilon / 9$. Let $x \in\left[x_{0}, x_{1}\right]$. Since $F(x)$ is between $F\left(x_{0}\right)$ and $F\left(x_{1}\right), a_{0} F(x)+b_{0}$ is between $a_{0} F\left(x_{0}\right)+b_{0}$ and $a_{0} F\left(x_{1}\right)+b_{0}$. We have

$$
\begin{aligned}
& \left|a_{0} F(x)+b_{0}-x\right| \\
& \leq\left|\left(a_{0} F(x)+b_{0}\right)-\left(a_{0} F\left(x_{0}\right)+b_{0}\right)\right| \\
& \quad+\left|a_{0} F\left(x_{0}\right)+b_{0}-x_{0}\right|+\left|x_{0}-x\right| \\
& \leq\left|\left(a_{0} F\left(x_{1}\right)+b_{0}\right)-\left(a_{0} F\left(x_{0}\right)+b_{0}\right)\right|+0+\frac{\varepsilon}{3} \\
& \leq\left|a_{0} F\left(x_{1}\right)+b_{0}-x_{1}\right|+\left|x_{1}-x_{0}\right| \\
& \quad+\left|x_{0}-\left(a_{0} F\left(x_{0}\right)+b_{0}\right)\right|+\frac{\varepsilon}{3} \\
& <\frac{\varepsilon}{9}+\frac{\varepsilon}{3}+0+\frac{\varepsilon}{3} \leq \varepsilon .
\end{aligned}
$$

In the second step, we can find real numbers $a_{1}, b_{1}$ such that the set $E \cup\left\{a_{0}, a_{1}\right\}$ is linearly independent over $\mathbb{Q}, a_{1} F\left(x_{1}\right)+$ 
$b_{1}=a_{0} F\left(x_{1}\right)+b_{0}$, and $\left|a_{1} F\left(x_{2}\right)+b_{1}-x_{2}\right|<\varepsilon / 9$. Let $x \in\left[x_{1}, x_{2}\right]$. Since $F(x)$ is between $F\left(x_{1}\right)$ and $F\left(x_{2}\right)$, then $a_{1} F(x)+b_{1}$ is between $a_{1} F\left(x_{1}\right)+b_{1}$ and $a_{1} F\left(x_{2}\right)+b_{1}$. We have

$$
\begin{aligned}
\left|a_{1} F(x)+b_{1}-x\right| & \mid \\
\leq & \left|\left(a_{1} F(x)+b_{1}\right)-\left(a_{1} F\left(x_{1}\right)+b_{1}\right)\right| \\
& +\left|a_{1} F\left(x_{1}\right)+b_{1}-x_{1}\right|+\left|x_{1}-x\right| \\
\leq & \left|\left(a_{1} F\left(x_{2}\right)+b_{1}\right)-\left(a_{1} F\left(x_{1}\right)+b_{1}\right)\right|+\frac{\varepsilon}{9}+\frac{\varepsilon}{3} \\
\leq & \left|a_{1} F\left(x_{2}\right)+b_{1}-x_{2}\right|+\left|x_{2}-x_{1}\right| \\
& +\left|x_{1}-\left(a_{1} F\left(x_{1}\right)+b_{1}\right)\right|+\frac{\varepsilon}{9}+\frac{\varepsilon}{3} \\
< & \frac{\varepsilon}{9}+\frac{\varepsilon}{3}+\frac{\varepsilon}{9}+\frac{\varepsilon}{3}+\frac{\varepsilon}{9}=\varepsilon .
\end{aligned}
$$

After $n$ steps, the construction is complete.

2.2. Main Theorem. Let $F:[a, b] \rightarrow \mathbb{R}$ be a continuous function. We consider the following operation on $F$. Let $a=$ $x_{0}<x_{1}<\cdots<x_{n}=b$ be a partition of $[a, b]$. Let $E_{F}$ : $[a, b] \rightarrow \mathbb{R}$ be such that $L_{F}(x)=f_{i}(F(x))$ for $x_{i} \leq x \leq x_{i+1}$, $f_{i}$ is exponential-like and $L_{F}$ is continuous. We say that $L_{F}$ is a continuous piecewise exponential-like transformation of $F$.

We say that a family $\mathscr{F}$ of continuous functions defined on compact intervals is flexible, provided

(1) $\mathscr{F}$ consists of nowhere constant functions;

(2) there is $f \in \mathscr{F}$ with $f \in C[0,1]$ and $f(0) \leq f(x) \leq$ $f(1)$ for $x \in[0,1]$

(3) $E_{f} \in \mathscr{F}$ for every $f \in \mathscr{F}$ and for any of its continuous piecewise exponential-like transformation $E_{f}$.

From now on we assume that $\mathscr{F}$ is flexible.

Theorem 8. $\mathscr{F} \cap C[0,1]$ is densely c-strongly algebrable in $C[0,1]$.

Proof. Let $F \in \mathscr{F}$ be such that $F \in C[0,1]$ and $F(0) \leq F(x) \leq$ $F(1)$ for any $x \in[0,1]$. Using Lemma 7 for $\varepsilon=1 / 2$ and $E=\emptyset$, we find a partition $0=x_{0}^{1}<x_{1}^{1}<\cdots<x_{n_{1}}^{1}=1$ of the unit interval and a continuous function $F_{1}$ such that

(i) $\left\|F_{1}-\mathrm{id}\right\|_{\text {sup }}<\varepsilon$;

(ii) $F_{1}(x)=a_{k}^{1} F(x)+b_{k}^{1}$ for $x_{k}^{1} \leq x \leq x_{k+1}^{1}, k=$ $0,1, \ldots, n_{1}-1$

(iii) the set $\left\{a_{0}^{1}, a_{1}^{1}, \ldots, a_{n_{1}-1}^{1}\right\}$ is linearly independent over Q.

In the next step we use Lemma 7 for $\varepsilon=1 / 4$ and $E=$ $\left\{a_{0}^{1}, a_{1}^{1}, \ldots, a_{n_{1}-1}^{1}\right\}$, and we find a refinement $0=x_{0}^{2}<x_{1}^{2}<$ $\cdots<x_{n_{2}}^{2}=1$ of the partition $0=x_{0}^{1}<x_{1}^{1}<\cdots<x_{n_{1}}^{1}=1$ and a continuous function $F_{2}$ such that

(i) $\left\|F_{2}-\mathrm{id}\right\|_{\text {sup }}<\varepsilon$;

(ii) $F_{2}(x)=a_{k}^{2} F(x)+b_{k}^{2}$ for $x_{k}^{2} \leq x \leq x_{k+1}^{2}, k=$ $0,1, \ldots, n_{2}-1$ (iii) the set $\left\{a_{0}^{1}, a_{1}^{1}, \ldots, a_{n_{1}-1}^{1}\right\} \cup\left\{a_{0}^{2}, a_{1}^{2}, \ldots, a_{n_{2}-1}^{2}\right\}$ is linearly independent over $\mathbb{Q}$ and so forth.

Inductively we define $F_{1}, F_{2}, \ldots$ Let $E=\bigcup_{k=1}^{\infty}\left\{a_{0}^{k}, a_{1}^{k}, \ldots\right.$, $\left.a_{n_{k}-1}^{k}\right\}$. By the construction, $E$ is linearly independent over $\mathbb{Q}$. We extend $E$ to a linearly independent set $H$ over $\mathbb{Q}$ of cardinality $c$. We may assume that there is $\left\{h_{n}: n \in \mathbb{N}\right\} \subset$ $H \backslash E$ with $h_{n} \rightarrow 0$. By the assumption, $\left\{\exp \circ F_{p}: p \in\right.$ $\mathbb{N}\} \cup\{\exp \circ(r F): r \in H \backslash E\} \subseteq \mathscr{F}$. Let $P$ be a polynomial in $m$ variables without a constant term. Consider a function $g=$ $P\left(e^{F_{1}}, \ldots, e^{F_{p}}, e^{r_{p+1} F}, \ldots, e^{r_{n} F}\right)$. Then, $g$ restricted to $\left[x_{l}^{p}, x_{l+1}^{p}\right]$ is of the form

$$
\begin{array}{r}
\sum_{i=1}^{m} c_{i} \exp \left(F ( x ) \left(d_{1} k_{i 1}+d_{2} k_{i 2}+\cdots+d_{p} k_{i p}\right.\right. \\
\left.\left.+r_{p+1} k_{i p+1}+\cdots+r_{n} k_{i n}\right)\right),
\end{array}
$$

where $d_{1}, \ldots, d_{p} \in E, r_{p+1}, \ldots, r_{n} \in H \backslash E$ are pairwise distinct and the vectors of integers $\left[k_{i 1}, k_{i 2}, \ldots, k_{i n}\right]$ are pairwise distinct. Therefore, the numbers $d_{1} k_{i 1}+d_{2} k_{i 2}+\cdots+d_{p} k_{i p}+$ $r_{p+1} k_{i p+1}+\cdots+r_{n} k_{i n}, i=1, \ldots, m$, are distinct as well. Thus, the mapping

$$
\begin{array}{r}
x \longmapsto \sum_{i=1}^{m} c_{i} \exp \left(F ( x ) \left(d_{1} k_{i 1}+d_{2} k_{i 2}+\cdots+d_{p} k_{i p}\right.\right. \\
\left.\left.+r_{p+1} k_{i p+1}+\cdots+r_{n} k_{i n}\right)\right)
\end{array}
$$

is a continuous exponential-like transformation of $F$ on $\left[x_{l}^{p}, x_{l+1}^{p}\right]$. Since $\mathscr{F}$ is closed under continuous piecewise exponential-like transformations, $g \in \mathscr{F}$.

This shows that the algebra $\mathscr{A}$ generated by $\left\{\exp \circ F_{p}\right.$ : $p \in \mathbb{N}\} \cup\{\exp \circ(r F): r \in H \backslash E\}$ is a free algebra of c generators. To see that $\mathscr{A}$ is dense in $C[0,1]$, note that the sequence $\exp \left(F_{1}\right), \exp \left(F_{2}\right), \ldots$ tends to $x \mapsto \exp (x)$, and therefore $\mathscr{A}$ separates the points of $[0,1]$. Moreover, note that $\lim _{n \rightarrow \infty} \exp \left(h_{n} F\right)=1$, which means that the closure of $\mathscr{A}$ contains all constant functions. Using Stone-Weierstrass theorem, we obtain the assertion.

2.3. Applications. (1) We say that a continuous function $F$ : $[a, b] \rightarrow \mathbb{R}$ is nowhere Hölder, provided that for any $x \in$ $[a, b]$ and any $\alpha \in(0,1]$

$$
\limsup _{y \rightarrow x} \frac{|F(x)-F(y)|}{|x-y|^{\alpha}}=\infty .
$$

Let us denote the set of all nowhere Hölder functions by $\mathcal{N} \mathscr{H}$. It was proved in [14] that $f \circ F \in \mathscr{N} \mathscr{H}$ for any nonconstant analytic function $f: \mathbb{R} \rightarrow \mathbb{R}$ and any $F \in$ $\mathscr{N} \mathscr{H}$. It can be easily seen that if $F:[a, b] \rightarrow \mathbb{R}$ and $F^{\prime}:[b, c] \rightarrow \mathbb{R}$ are nowhere Hölder with $F(b)=F^{\prime}(b)$, then $F \cup F^{\prime}:[a, c] \rightarrow \mathbb{R}$ is also nowhere Hölder. Therefore, $\mathcal{N} \mathscr{H}$ is closed under taking continuous piecewise exponential-like transformations. Clearly $\mathscr{N} \mathscr{H}$ does not contain a function which is constant on some open interval.

Now, we prove that condition (2) in definition of flexibility is fulfilled. Let $F \in \mathscr{N} \mathscr{H} \cap C[0,1]$. We may assume that 
$F(0) \leq F(1)$ (otherwise, consider $-F$ which is also nowhere Hölder). If $F(0) \leq F(x) \leq F(1)$ for $x \in[0,1]$, then we are done. Otherwise, find a maximizer $x_{0} \in(0,1)$ of $F$. Then, $F(x) \leq F\left(x_{1}\right)$ for $x \in\left[0, x_{0}\right]$. If $F(0) \leq F(x) \leq F\left(x_{0}\right)$ for $x \in\left[0, x_{0}\right]$, then an affine transformation $t \mapsto F\left(t / x_{0}\right)$ of $\left.F\right|_{\left[0, x_{0}\right]}$ fulfills condition (2) in the definition of a flexible family. Otherwise, find a minimizer $x_{1} \in\left(0, x_{0}\right)$ of $\left.F\right|_{\left[0, x_{0}\right]}$. Then, $F\left(x_{1}\right) \leq F(x) \leq F\left(x_{0}\right)$ for $x \in\left[x_{1}, x_{0}\right]$. Then, an affine transformation $t \mapsto F\left(t /\left(x_{0}-x_{1}\right)-x_{1} /\left(x_{0}-x_{1}\right)\right)$ of $\left.F\right|_{\left[x_{1}, x_{0}\right]}$ fulfills condition (2) in the definition of a flexible family. This argument will hold also for the next families.

Finally, by Theorem 8 , the set of all nowhere Hölder functions in $C[0,1]$ is densely c-strongly algebrable.

(2) We say that a continuous function $f:[a, b] \rightarrow \mathbb{R}$ is Bruckner-Garg of rank $k \in \mathbb{N}$ (shortly $f \in \mathscr{B}_{k}$ ), provided that there exists a countable set $A \subseteq(\min f, \max f)$ with the property that for all $x \in A$ the preimage $f^{-1}(\{x\})$ is a union of a Cantor set with at most $k$ many isolated points and for all $x \in(\min f, \max f) \backslash A$ the preimage $f^{-1}(\{x\})$ is a Cantor set. A function $f$ is Bruckner-Garg (shortly $f \in \mathscr{B} \mathscr{G}_{\omega}$ ), provided it is Bruckner-Garg of rank $k$ for some $k \in \mathbb{N}$. BrucknerGarg functions of rank 1 were investigated in [17], where it was shown that $\mathscr{B} \mathscr{G}_{1}$ is residual in $C[0,1]$. By [14, Theorem 4.13] we can easily conclude that $\mathscr{B} \mathscr{G}_{\omega}$ is flexible and hence it is densely $\boldsymbol{c}$-strongly algebrable.

(3) Let $C M m([0,1])$ be the set of all continuous functions such that both sets of their proper local minima and maxima are dense in $[0,1]$. Using a similar argument to that in [12], one can prove that the set of all functions from $\operatorname{CMm}([0,1])$ is flexible and thereby it is densely $\boldsymbol{c}$-strongly algebrable.

(4) Denote by $D N M$ the set of all functions in $C[0,1]$ which are nowhere monotonic and differentiable in all but finitely many points; see [18]. It can be shown in a standard way that $D N M$ is flexible; thus, it is densely $\mathfrak{c}$-strongly algebrable.

\section{Closed Algebrability}

Aron et al. posed the following problem [19, Problem 4.1]: Characterize when there exists a closed infinite dimensional algebra of functions with a particular "strange" property. Among the classes considered by the authors, there was the family of everywhere surjective functions $f: \mathbb{C} \rightarrow \mathbb{C}$. In the space $\mathbb{C}^{X}$ or $\mathbb{R}^{X}, X \neq \emptyset$, we consider two natural topologies, namely, the topology $\tau_{p}$ of pointwise convergence-the weakest topology in which each projection is continuousand the topology $\tau_{u}$ of uniform convergence. We will show that the $\tau_{p}$-closure of any nontrivial algebra contains a twovalued function (some characteristic function). Moreover, we will give a sufficient condition for the existence of a closed algebra inside $\mathscr{F} \cup\{0\}$ of $2^{\mathfrak{C}}$ generators.

The following proposition shows that if $\mathscr{A}$ is a $\tau_{p}$-closed nontrivial algebra, then $\mathscr{A}$ contains a two-valued function.

Proposition 9. Let $\mathscr{A}$ be a subalgebra of $\mathbb{C}^{X}$ or $\mathbb{R}^{X}$. Then for any $f \in \mathscr{A}$ the characteristic function $\chi_{S}$ of $S:=\{x \in X$ : $f(x) \neq 0\}$ is in $\mathrm{cl}_{\tau_{p}}(\mathscr{A})$.
Proof. Let $f \in \mathscr{A} \subset \mathbb{R}^{X}$. Let $g=\chi_{S}$ be the characteristic function of $S$. Take any $x_{1}, \ldots, x_{n} \in X$ and $\varepsilon>0$. Let $V=$ $\left\{h \in \mathbb{R}^{X}:\left|h\left(x_{i}\right)-g\left(x_{i}\right)\right|<\varepsilon\right.$ for $\left.i=1, \ldots, n\right\}$. We need to show that $\mathscr{A} \cap V \neq \emptyset$. Let $Y=\left\{f\left(x_{i}\right): f\left(x_{i}\right) \neq 0, i=\right.$ $1, \ldots, n\}=\left\{y_{1}, \ldots, y_{k}\right\}$. Put

$$
P(y)=\sum_{j=1}^{k} \frac{y}{y_{j}} \prod_{i \neq j} \frac{y-y_{i}}{y_{j}-y_{i}} .
$$

Then, $P$ is a polynomial without a constant term such that $P\left(y_{j}\right)=1$ for any $j=1, \ldots, k$. If $f\left(x_{i}\right)=0$, then $P(f)\left(x_{i}\right)=0$. Since $x_{i} \notin S, f\left(x_{i}\right)=g\left(x_{i}\right)$. If $f\left(x_{i}\right) \neq 0$, then $y_{j}=f\left(x_{i}\right)$ for some $j$ and $P(f)\left(x_{i}\right)=P\left(y_{j}\right)=1=g\left(x_{i}\right)$. This shows that $P(f) \in \mathscr{A} \cap V$.

By $\mathscr{E} \mathcal{S}(\mathbb{C})$, we denote the family of all everywhere surjective functions $f: \mathbb{C} \rightarrow \mathbb{C}$, that is, functions which map any nonempty open subset of $\mathbb{C}$ onto $\mathbb{C}$. This family appeared at first in terms of algebrability in [7]. By $\mathscr{P} \mathscr{E} \mathcal{S}(\mathbb{C})$, we denote the family of all perfectly everywhere surjective functions $f: \mathbb{C} \rightarrow \mathbb{C}$, that is, functions which map any perfect subset of $\mathbb{C}$ onto $\mathbb{C}$. It was proved in [20] that $\mathscr{P} \mathscr{E} \mathcal{S}(\mathbb{C})$ is $2^{\mathrm{c}}$-strongly algebrable. Since $\mathscr{P} \mathscr{E} \mathcal{S}(\mathbb{C}) \subset \mathscr{E} \mathcal{S}(\mathbb{C}), \mathscr{E} \mathcal{S}(\mathbb{C})$ is $2^{\mathrm{c}}$-strongly algebrable too. Let $\mathscr{D}$ stand for the family of all nonconstant Darboux functions. Since any nonconstant Darboux function attains $\mathfrak{c}$ many values, we obtain the following.

Corollary 10. $\mathscr{D} \cup\{0\}$ does not contain a nontrivial closed algebra. In particular, the set $\mathscr{E} \mathcal{S}(\mathbb{C})$ of all everywhere surjective functions is not 1- $\tau_{p}$-closed-algebrable.

Proposition 9 says that any $\tau_{p}$-closed algebra contains two-valued functions. The next step is searching for large $\tau_{p^{-}}$ closed algebras in those consisting of functions with a finite range. Note that $\left\{f \in \mathbb{R}^{\mathbb{R}}: f\right.$ has a finite range $\}$ is an algebra of cardinality $2^{\mathrm{c}}$. However, the following shows that it does not contain a large $\tau_{p}$-closed (even $\tau_{u}$-closed) algebra.

Theorem 11. Let $\mathscr{A}$ be an algebra consisting of functions with finite ranges. Then

(i) if $\mathscr{A}$ is finitely generated, then $\mathscr{A}$ is $\tau_{p}$-closed;

(ii) if $\mathscr{A}$ is not finitely generated, then $\mathscr{A}$ is not $\tau_{u}$-closed (in particular, it is not $\tau_{p}$-closed).

Proof. (i) Assume that $\mathscr{A}$ is generated by $f_{1}, \ldots, f_{n}$. Since each $f_{i}$ has a finite range, we can write

$$
f_{i}=\sum_{j=1}^{k_{i}} c_{i j} \chi_{A_{i j}},
$$

where $c_{i 1}, \ldots, c_{i k_{i}}$ are distinct and $A_{i 1}, \ldots, A_{i k_{i}}$ is a partition of $\mathbb{R}$. Let $\mathscr{B}$ stand for all finite Boolean combinations of $\left\{A_{i j}\right.$ : $\left.i=1, \ldots, n, j=1, \ldots, k_{i}\right\}$. Clearly, any member of $\mathscr{A}$ is $\mathscr{B}$ measurable. Let $A \in \mathscr{B}$ be a nonempty atom of the algebra $\mathscr{B}$. Then, there are $j_{1}, \ldots, j_{n}$ such that $A=A_{1 j_{1}} \cap \cdots \cap A_{n j_{n}}$. For any $i=1, \ldots, n$, there is a polynomial $P_{i}$ such that $P_{i}\left(c_{i j_{i}}\right)=1$ and $P_{i}\left(c_{i j}\right) \leq 0$ for $j \neq j_{i}$. Then,

$$
P_{1}\left(f_{1}(x)\right)+\cdots+P_{n}\left(f_{n}(x)\right)>0 \Longleftrightarrow x \in A .
$$


Since $P_{1}\left(f_{1}\right)+\cdots+P_{n}\left(f_{n}\right)$ is constant on $A$ and has finitely many values, there is a polynomial $P$ such that $P\left(P_{1}\left(f_{1}\right)+\right.$ $\left.\cdots+P_{n}\left(f_{n}\right)\right)$ is a characteristic function of $A$. Therefore, any $\mathscr{B}$-measurable function is in $\mathscr{A}$. Since $\mathscr{B}$ is a $\sigma$-algebra of sets, the family of all $\mathscr{B}$-measurable functions is $\tau_{p^{-}}$ closed (a pointwise limit of $\mathscr{B}$-measurable functions is $\mathscr{B}$ measurable).

(ii) Assume now that $\mathscr{A}$ is not finitely generated. There are $f_{1}, f_{2}, \ldots \in \mathscr{A}$ which are algebraically independent. As before, $f_{i}=\sum_{j=1}^{k_{i}} c_{i j} \chi_{A_{i j}}$ and let $\mathscr{B}$ stand for the set of all finite Boolean combinations of $\left\{A_{i j}: i \in \mathbb{N}, j=1, \ldots, k_{i}\right\}$. Suppose that $\mathscr{B}$ is finite. Again, any characteristic function of an atom in $\mathscr{B}$ is an algebraic combination of finitely many $f_{i}$ 's. Therefore, there is $n \in \mathbb{N}$ such that any $\mathscr{B}$-measurable function $f$ is an algebraic combination of $f_{1}, \ldots, f_{n}$. This yields a contradiction. Therefore, $\mathscr{B}$ is infinite. Hence, we can find pairwise disjoint sets $A_{1}, A_{2}, \ldots \in \mathscr{B}$. Define $f_{n}=$ $\sum_{k=1}^{n}(1 / k) \chi_{A_{k}}$. Since $\chi_{A_{i}} \in \mathscr{A}$, each $f_{n}$ is in $\mathscr{A}$. Clearly, $f_{n}$ tends uniformly to $f=\sum_{k=1}^{\infty}(1 / k) \chi_{A_{k}} \notin \mathscr{A}$.

By $\mathscr{E} \mathscr{D} \mathscr{F}$, denote the family of all functions $f: \mathbb{R} \rightarrow \mathbb{R}$ which are everywhere discontinuous and $f(\mathbb{R})$ is finite. It was proved in [21] that $\mathscr{E} \mathscr{D} \mathscr{F}$ is $2^{\mathfrak{C}}$-algebrable. Immediately we obtain the following.

Corollary 12. $\mathscr{E} \mathscr{D} \mathscr{F} \cup\{0\}$ does not contain an infinitely generated $\tau_{u}$-closed algebra.

By Proposition 9 and Theorem 11, any infinitely generated $\tau_{p}$-closed algebra contains finite valued and countably valued functions. It turns out that there are large $\tau_{p}$-closed algebras of countably valued functions. Such construction, using the existence of large $\sigma$-independent family, will be used in the next theorem.

A family $\left\{A_{\alpha}: \alpha<\kappa\right\}$ of subsets of $Y$ is called $\sigma$ independent, if for every countable set $X \subset \kappa$ and every $\varepsilon: X \rightarrow\{0,1\}$

$$
\bigcap_{\alpha \in X} A_{\alpha}^{\varepsilon(\alpha)} \neq \emptyset
$$

where $A^{0}=A$ and $A^{1}=Y \backslash A$. By the Tarski theorem [22] there exists a $\sigma$-independent family on $\mathfrak{c}$ of cardinality $2^{\mathfrak{c}}$.

Theorem 13. There is a linear algebra $\mathscr{A} \subset \mathbb{R}^{\mathbb{R}}$ of $2^{\mathfrak{c}}$ generators such that for any function $f \in \mathrm{cl}_{\tau_{p}}(\mathscr{A}) \backslash\{0\}$ there is open set $U$ such that $f^{-1}(U)$ is a Bernstein set. In particular, if $\mathscr{F}$ is the family of all nonmeasurable functions (having no Baire property, nonmeasurable in the sense of Marczewski), then $\mathscr{F} \cup\{0\}$ contains a $\tau_{p}$-closed algebra of $2^{\mathfrak{c}}$ generators.

Proof. We use the method of independent Bernstein sets which was introduced in [21]. Let $\left\{B_{\alpha}: \alpha<\mathfrak{c}\right\}$ be a partition of $\mathbb{R}$ into $\mathfrak{c}$ many pairwise disjoint Bernstein sets. Let $\left\{A_{\xi}\right.$ : $\left.\xi<2^{\mathfrak{c}}\right\}$ be a $\sigma$-independent family on $\mathfrak{c}$. For any $\xi<2^{\mathfrak{c}}$, put $C_{\xi}=\bigcup\left\{B_{\alpha}: \alpha \in A_{\xi}\right\}$. Let $\mathscr{B}$ be the $\sigma$-algebra generated by $\left\{C_{\xi}: \xi<2^{\mathfrak{c}}\right\}$.

Let $\mathscr{A}$ be the linear algebra generated by $\left\{\chi_{C_{\xi}}: \xi<\right.$ $\left.2^{\mathfrak{c}}\right\}$. Then each function in $\mathscr{A}$ is a simple function of the form $\sum_{k=1}^{2^{n}} c_{k} \chi_{D_{k}}$, where $D_{k}$ are Boolean combinations of $C_{\xi_{1}}, \ldots, C_{\xi_{n}}$ for some distinct $\xi_{1}, \ldots, \xi_{n}<2^{\mathfrak{c}}$. If $f \in \mathrm{cl}_{\tau_{p}}(\mathscr{A}) \backslash$ $\{0\}$, then there are $f_{n} \in \mathscr{A}$ which tend pointwisely to $f$. Let $X \subset 2^{c}$ be the smallest set such that each $f_{n}$ is measurable with respect to $\sigma$-algebra $\mathscr{B}_{X}$ generated by $\left\{C_{\xi}: \xi \in X\right\}$. Clearly $X$ is countable. There is $\alpha<\mathfrak{c}$ which does not belong to any $A_{\xi}, \xi \in X$. Consequently, $B_{\alpha} \subset \bigcap_{\xi \in X} \mathbb{R} \backslash C_{\xi}$. Therefore, $\left.f_{n}\right|_{B_{\alpha}}=0$ and $\left.f\right|_{B_{\alpha}}=0$. Since $f$ is not the zero function, $f(x) \neq 0$ for some $x \in \mathbb{R}$. There is $\delta>0$ such that $f^{-1}(f(x)-\delta, f(x)+\delta)$ is disjoint with $f^{-1}(0)$. Since $f$ is $\mathscr{B}_{X^{-}}$ measurable, $f^{-1}(f(x)-\delta, f(x)+\delta)$ contains a Bernstein set of the form $\bigcap_{\xi \in X} C_{\xi}^{\varepsilon(\xi)}$ for some $\varepsilon: X \rightarrow\{0,1\}$. Finally, a set which contains a Bernstein set and is disjoint with some other Bernstein sets is also a Bernstein set.

Let $f \in \mathbb{R}^{X}$ (or $f \in \mathbb{C}^{X}$ ). Fix the partition $\left\{B_{\xi}: \xi<\kappa\right\}$ of $\mathbb{R}$ (or $\mathbb{C}$ ). By $V(f)$ we define the set

$$
\begin{gathered}
\left\{\left.\bigcup_{\xi<\kappa} t(\xi) f\right|_{B_{\xi}}: t \in \mathbb{R}^{\kappa}\right\}, \\
\left(\left\{\left.\bigcup_{\xi<\kappa} t(\xi) f\right|_{B_{\xi}}: t \in \mathbb{C}^{\kappa}\right\}, \text { resp. }\right) .
\end{gathered}
$$

Let $g_{1}, \ldots, g_{n} \in V(f)$ and let $P\left(y_{1}, \ldots, y_{n}\right)$ be a polynomial in $n$ variables. Let $t_{i}(\xi)$ be such that $\left.g_{i}\right|_{B_{\xi}}=\left.t_{i}(\xi) f\right|_{B_{\xi}}$. Then,

$$
\begin{aligned}
P & \left.\left(g_{1}, \ldots, g_{n}\right)\right|_{B_{\xi}} \\
& =P\left(\left.g_{1}\right|_{B_{\xi}}, \ldots,\left.g_{n}\right|_{B_{\xi}}\right) \\
& =P\left(\left.t_{1}(\xi) f\right|_{B_{\xi}}, \ldots,\left.t_{n}(\xi) f\right|_{B_{\xi}}\right)=\left.P^{\prime}(f)\right|_{B_{\xi}},
\end{aligned}
$$

where $P^{\prime}(y)=P\left(t_{1}(\xi) y, \ldots, t_{n}(\xi) y\right)$. Therefore, the algebra $\mathscr{A}(f)$ generated by $V(f)$ is of form

$$
\mathscr{A}(f)=\left\{\bigcup_{\xi<\kappa} f_{\xi}: f_{\xi} \in \mathscr{A}_{\xi}\right\},
$$

where $\mathscr{A}_{\xi}$ is a subalgebra of $\mathbb{C}^{B_{\xi}}$ generated by $\left.f\right|_{B_{\xi}}$.

Theorem 14. Assume that $\left.f\right|_{B_{\xi}}$ is unbounded for every $\xi<\kappa$. Then, $\mathscr{A}(f)$ is $\tau_{u}$-closed.

Proof. Note that $\tau_{u}$ is metrizable by the metric $d(g, h)=$ $\min \{1, \sup \{|g(x)-h(x)|: x \in \mathbb{C}\}\}$. To prove that $\mathscr{A}(f)$ is $\tau_{u}$-closed, take a sequence $\left(g_{n}\right)$ in $\mathscr{A}(f)$ tending with respect to $d$ to some function $g$. Fix $\xi<\kappa$. If $g$ is zero on $B_{\xi}$, then obviously $g \in \mathscr{A}_{\xi}$. Otherwise, $\left.g\right|_{B_{\xi}}$ is nonzero. Then, the sequence $\left(\left.g_{n}\right|_{B_{\xi}}\right)_{n \in \mathbb{N}}$ eventually consists of nonzero functions. Note that $\left.g_{n}\right|_{B_{\xi}}=\left.P_{n}(f)\right|_{B_{\xi}}$ for some nonzero polynomials $P_{n}$ in one variable. By the assumption $f\left(B_{\xi}\right)$ is unbounded. Note that the sequence $P_{n}: f\left(B_{\xi}\right) \rightarrow \mathbb{C}$ is a Cauchy sequence with respect to $d(g, h)=\min \left\{1, \sup \left\{|g(y)-h(y)|: y \in f\left(B_{\xi}\right)\right\}\right\}$ for $g, h \in \mathbb{C}^{f\left(B_{\xi}\right)}$. Since $f\left(B_{\xi}\right)$ is unbounded, then, for distinct 
polynomials in one variable $P, Q: f\left(B_{\xi}\right) \rightarrow \mathbb{C}$ without constant term, we have $\sup \left\{|P(y)-Q(y)|: y \in f\left(B_{\xi}\right)\right\}=\infty$. Therefore, the sequence $\left(P_{n}\right)$ is eventually constant and equal to some polynomial $P$. Thus, $\left.g\right|_{B_{\xi}}=\left.P(f)\right|_{B_{\xi}} \in \mathscr{A}_{\xi}$.

Corollary 15. There exists a $\tau_{u}$-closed algebra $\mathscr{A}$ of cardinality $2^{\mathfrak{c}}$ and hence $2^{\mathrm{c}}$-generated, such that $\mathscr{A} \backslash\{0\}$ consists of perfectly everywhere surjective functions.

Proof. Let $\left\{B_{\xi}: \xi<\mathfrak{c}\right\}$ be a decomposition of $\mathbb{C}$ into $\mathfrak{c}$ many Bernstein sets. For any $\xi<\mathfrak{c}$, let $f_{\xi}: B_{\xi} \rightarrow \mathbb{C}$ be a free generator such that algebra generated by $f_{\xi}$ consists of perfectly everywhere surjective functions; the existence of such a function was proved in [20]. Put $f=\bigcup_{\xi<c} f_{\xi}: \mathbb{C} \rightarrow$ $\mathbb{C}$. Then, $\mathscr{A}(f)$ is the desired algebra.

For a sequence $x \in \ell_{\infty}$, put $\operatorname{LIM}(x)=\left\{y \in \mathbb{R}: x\left(n_{k}\right) \rightarrow\right.$ $y$ for some increasing $\left.\left(n_{k}\right)_{k \in \mathbb{N}}\right\}$. It was proved in [8] that the set of $x \in \ell_{\infty}$ for which $\operatorname{LIM}(x)$ is homeomorphic to the Cantor set is strongly $\mathfrak{c}$-algebrable and comeager. We complete this result with the following.

Theorem 16. The set of those $x \in \ell_{\infty}$, for which $\operatorname{LIM}(x)$ is homeomorphic to the Cantor set, does not contain any nontrivial closed algebra.

Proof. Let $\mathscr{A}$ be an algebra such that for any $x \in \mathscr{A} \backslash\{0\}$ the set of limit points $\operatorname{LIM}(x)$ is homeomorphic to the Cantor set. Fix nonzero $x \in \mathscr{A}$ and let $C=\operatorname{LIM}(x)$. There is a continuous function $f:[\min C, \max C] \rightarrow[0,1]$ such that $f(C)=[0,1]$. Let $\left(P_{n}\right)$ be a sequence of polynomials, tending uniformly to $f$. It is evident that $P_{n}(x)$ tends in $\ell_{\infty}$ to some $y$ with $\operatorname{LIM}(y)=[0,1]$. Since $[0,1]$ is not homeomorphic to $C$, the algebra $\mathscr{A}$ cannot be closed.

\section{Conflict of Interests}

The authors declare that there is no conflict of interests regarding the publication of this paper.

\section{Acknowledgment}

Szymon Głąb has been supported by the National Science Centre, Poland, Grant no. DEC-2012/07/D/ST1/02087.

\section{References}

[1] T. Radaković, "Über Darbouxsche und stetige Funktionen," Monatshefte für Mathematik und Physik, vol. 38, no. 1, pp. 117$122,1931$.

[2] B. Levine and D. Milman, "On linear sets in space C consisting of functions of bounded variation," Communications de l'Institut des Sciences Mathématiques et Méchaniques de l'Université de Kharkoff, vol. 16, pp. 102-105, 1940.

[3] V. I. Gurariǔ, "Subspaces and bases in spaces of continuous functions," Doklady Akademii Nauk SSSR, vol. 167, pp. 971-973, 1966 (Russian).

[4] V. I. Gurariü, "Linear spaces composed of everywhere nondifferentiable functions," Comptes Rendus de l'Académie Bulgare des Sciences, vol. 44, no. 5, pp. 13-16, 1991.
[5] R. M. Aron, V. I. Gurariy, and J. B. Seoane, "Lineability and spaceability of sets of functions on $\mathbb{R}$," Proceedings of the American Mathematical Society, vol. 133, no. 3, pp. 795-803, 2005.

[6] R. M. Aron, D. Pérez-García, and J. B. Seoane-Sepúlveda, "Algebrability of the set of non-convergent Fourier series," Studia Mathematica, vol. 175, no. 1, pp. 83-90, 2006.

[7] R. M. Aron and J. B. Seoane-Sepúlveda, "Algebrability of the set of everywhere surjective functions on $\mathbb{C}$," Bulletin of the Belgian Mathematical Society-Simon Stevin, vol. 14, no. 1, pp. 25-31, 2007.

[8] A. Bartoszewicz and S. Głąb, "Strong algebrability of sets of sequences and functions," Proceedings of the American Mathematical Society, vol. 141, no. 3, pp. 827-835, 2013.

[9] K. C. Ciesielski, J. L. Gámez-Merino, D. Pellegrino, and J. B. Seoane-Sepúlveda, "Lineability, spaceability, and additivity cardinals for Darboux-like functions," Linear Algebra and Its Applications, vol. 440, pp. 307-317, 2014.

[10] F. Bayart and L. Quarta, "Algebras in sets of queer functions," Israel Journal of Mathematics, vol. 158, pp. 285-296, 2007.

[11] F. Bastin, J. A. Conejero, C. Esser, and J. B. Seoane-Sepúlveda, "Algebrability and nowhere Gevrey differentiability," Israel Journal of Mathematics, 2014.

[12] D. García, B. C. Grecu, M. Maestre, and J. B. Seoane-Sepúlveda, "Infinite dimensional Banach spaces of functions with nonlinear properties," Mathematische Nachrichten, vol. 283, no. 5, pp. 712-720, 2010.

[13] M. Balcerzak, A. Bartoszewicz, and M. Filipczak, "Nonseparable spaceability and strong algebrability of sets of continuous singular functions," Journal of Mathematical Analysis and Applications, vol. 407, no. 2, pp. 263-269, 2013.

[14] A. Bartoszewicz, M. Bienias, M. Filipczak, and S. Głąb, "Strong c-algebrability of strong Sierpiński-Zygmund, smooth nowhere analytic and other sets of functions," Journal of Mathematical Analysis and Applications, vol. 412, no. 2, pp. 620-630, 2014.

[15] S. Głąb, "Local and global monotonicity" Real Analysis Exchange, vol. 27, no. 2, pp. 765-772, 2001.

[16] A. M. Bruckner, Differentiation of Real Functions, vol. 659 of Lecture Notes in Mathematics, Springer, Berlin, Germany, 1978.

[17] A. M. Bruckner and K. M. Garg, "The level structure of a residual set of continuous functions," Transactions of the American Mathematical Society, vol. 232, pp. 307-321, 1977.

[18] R. M. Aron, F. J. García-Pacheco, D. Pérez-García, and J. B. Seoane-Sepúlveda, "On dense-lineability of sets of functions on $\mathbb{R}$," Topology, vol. 48, no. 2-4, pp. 149-156, 2009.

[19] R. M. Aron, J. A. Conejero, A. Peris, and J. B. Seoane-Sepúlveda, "Sums and products of bad functions," in Function Spaces, vol. 435 of Contemporary Mathematics, pp. 47-52, American Mathematical Society, Providence, RI, USA, 2007.

[20] A. Bartoszewicz, S. Głąb, and A. Paszkiewicz, "Large free linear algebras of real and complex functions," Linear Algebra and Its Applications, vol. 438, no. 9, pp. 3689-3701, 2013.

[21] A. Bartoszewicz, M. Bienias, and S. Głąb, "Independent Bernstein sets and algebraic constructions," Journal of Mathematical Analysis and Applications, vol. 393, no. 1, pp. 138-143, 2012.

[22] A. Tarski, “Ideale in vollständige Mengenkörpern. I," Fundamenta Mathematicae, vol. 32, pp. 45-63, 1939. 


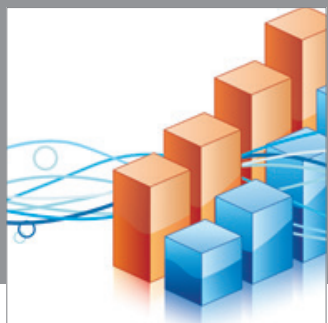

Advances in

Operations Research

mansans

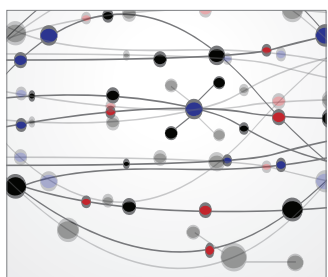

The Scientific World Journal
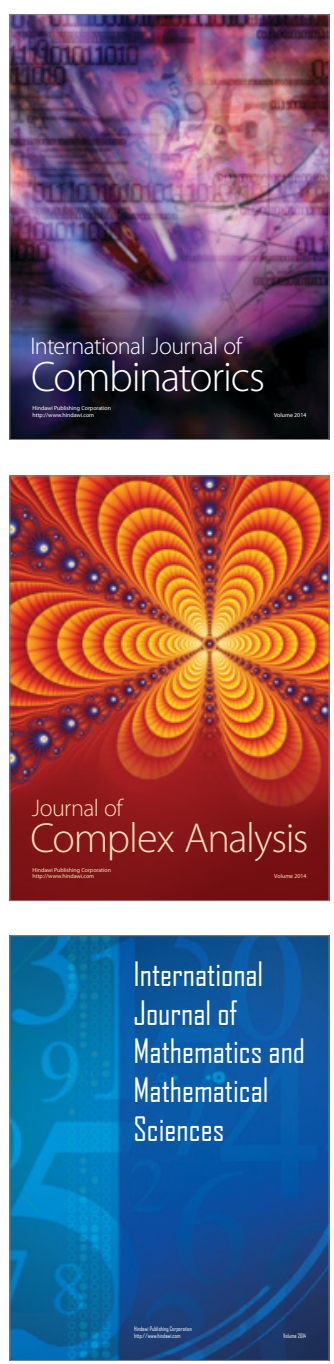
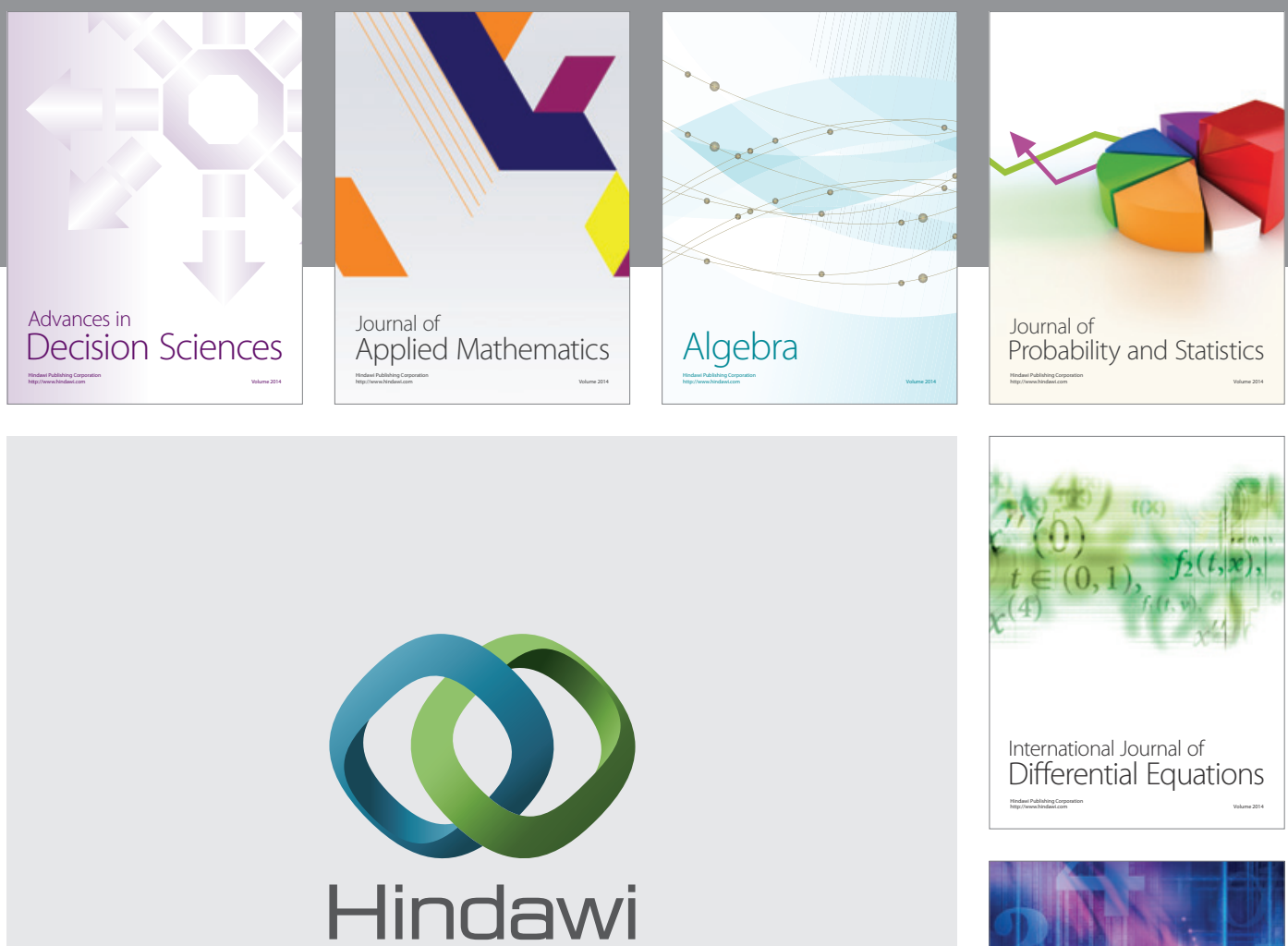

Submit your manuscripts at http://www.hindawi.com
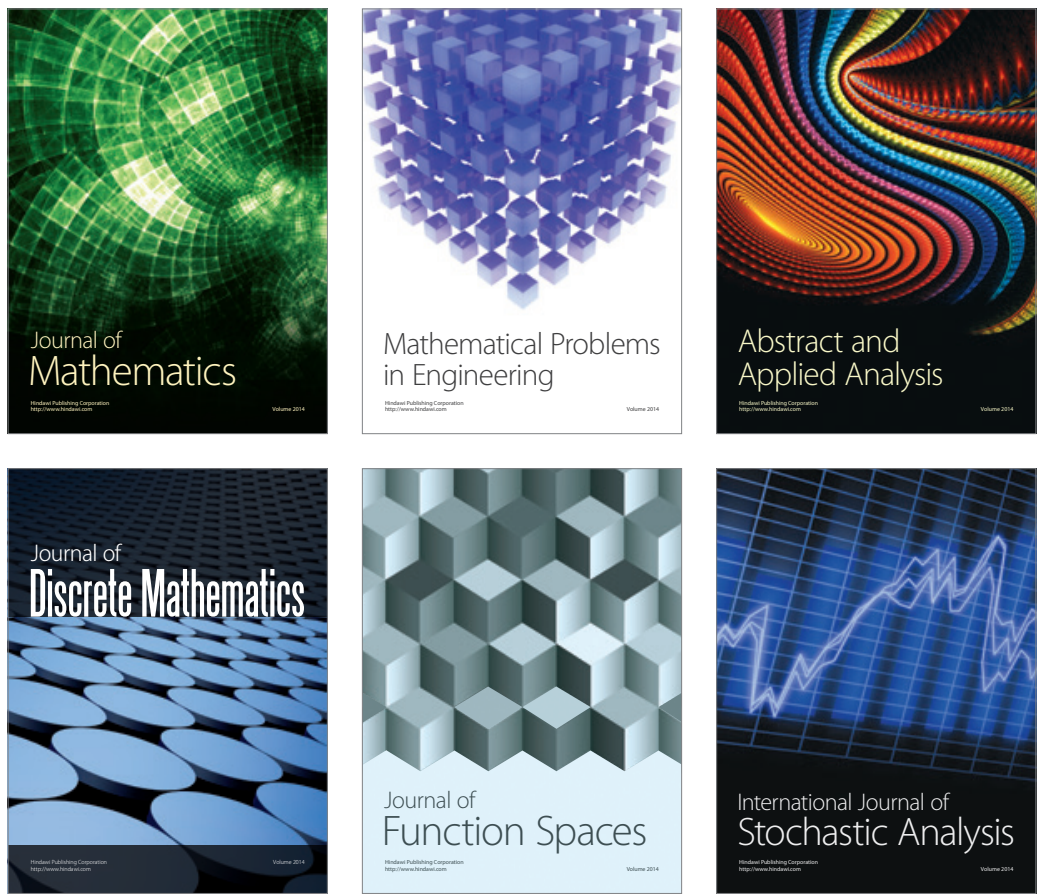

Journal of

Function Spaces

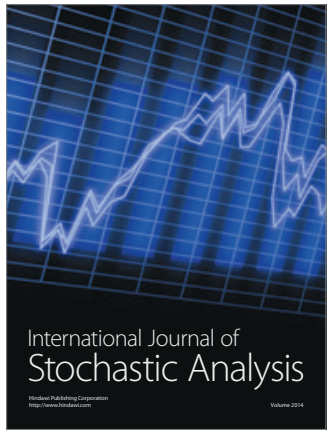

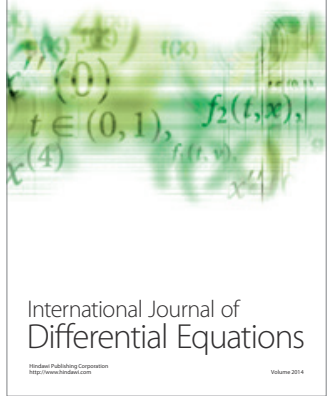
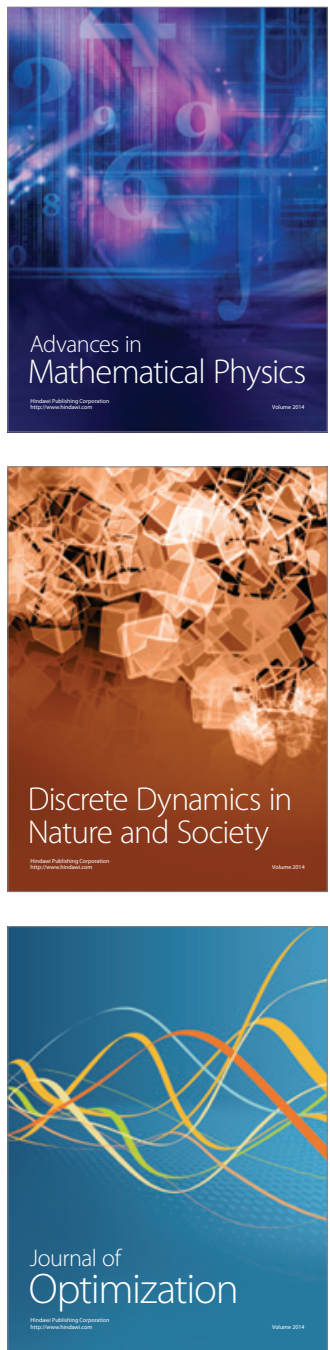